\title{
ON THE ALMOST SURE CENTRAL LIMIT THEOREM FOR ARX PROCESSES IN ADAPTIVE TRACKING
}

\author{
BERNARD BERCU AND VICTOR VAZQUEZ
}

\begin{abstract}
The goal of this paper is to highlight the almost sure central limit theorem for martingales to the control community and to show the usefulness of this result for the system identification of controllable $\operatorname{ARX}(p, q)$ process in adaptive tracking. We also provide strongly consistent estimators of the even moments of the driven noise of a controllable $\operatorname{ARX}(p, q)$ process as well as quadratic strong laws for the average costs and estimation errors sequences. Our theoretical results are illustrated by numerical experiments.
\end{abstract}

\section{INTRODUCTION}

Zadeh [32] introduced the concept of system identification as the determination, on the basis of input and output, of a system within a specified class of systems to which the system under test is equivalent. Hence, any theoretical result which leads us to make such determination in a more precise way will be a step into the spirit of Zadeh's definition. In order to track some of the most relevant results in system identification, we may find exhaustive and very useful reviews summarizing the most important contributions in this area of applied mathematics. To the best of our knowledge, the most relevant of them are the survey of Aström and Eykhoff [1], the excellent book of Aström and Wittenmark [2], the work of Ljung [27] devoted to adaptive tracking in system identification, and the beautiful and captivating bookchapter of Gevers [20] which deals with the recent developments in identification theory. We also refer the reader to 23 . for an overview of basic research on model selection approaches for linear systems and to [29] for Kernel methods in system identification, machine learning and functional estimation.

Since the pioneer works on system identification and adaptive control, there has been a great deal of activity from the control community on the theoretical aspects as well as on the practical applications. Recently, Cho et al. [17] proposed a new parameter estimation method in the framework of composite model reference adaptive control, in order to improve parameter estimation without persistent excitation. Heydari [21] investigated the stability of adaptive optimal control using value iteration, initiated from a stabilizing control policy. Jaramillo et al. [24] presented an adaptive control framework for compensation of uncertainties and perturbations that satisfy the matching condition on a class of nonlinear dynamic systems. Moreover, Zhu et al. [34] proposed an adaptive model predictive control for unconstrained

2010 Mathematics Subject Classification. Primary: 62G05 Secondary: 93C40, 60F05.

Key words and phrases. ARX process; Controllability; Almost sure central limit theorem; least squares estimation. 
discrete-time linear systems with parametric uncertainties We also refer the reader to Gao et al. [19] who investigated the problem of adaptive tracking control for a class of stochastic uncertain nonlinear systems in the presence of input saturation, to Zhao et al. 33 who studied the adaptive control for linear systems with set-valued observations in order to track a given periodic target, and to Tao et al. [31] for the higher-order tracking properties of model reference adaptive control systems.

Bercu et al. [6, 7] investigated the asymptotic behavior of the least squares estimator for ARX process in adaptive tracking [2]. More precisely, a new notion of strong controllability for multidimensional ARX processes was proposed in [6]. In addition, via a persistently excited version of the adaptive control, it has been shown in [7] how to avoid this strong controllability condition. In the scalar framework, a serial correlation noise was considered in [8] for ARX processes. The asymptotic behaviour of the least squares estimator was analyzed together with the almost sure convergence of the Durbin-Watson statistics as well as its asymptotic normality. It led us to proposed a bilateral statistical test for testing whether or not the serial correlation parameter is equal to some non zero fixed value. Finally, in [9], the introduction of a persistent excitation in the Ästrom and Wittenmark adaptive control led us to explore a statistical test for detecting the presence of a serial correlated noise together with the asymptotics of the least squares estimator and of the Durbin-Watson statistics.

The primary goal of this paper is to highlight the almost sure central limit theorem (ASCLT) for martingales to the control community and to show the usefulness of this result for the system identification of a controllable $\operatorname{ARX}(p, q)$ process in adaptive tracking. The ASCLT has been widely investigated in stochastic approximation theory [5, 13, 28] and in statistics [11, 22]. On the one hand, a large literature is available on the ASCLT for sums of independent random variables [12, 25, 30, 10]. On the other hand, it is also possible to find many references on the ASCLT for martingales [4, 5, 15, 16, 26].

Surprisingly, the deep impact of the ASCLT has not deeply reached to the control community. To the best of our knowledge, no reference is available in the engineering literature dealing the ASCLT. Hence, the aim of this paper is to show how the ASCLT for martingales could provide interesting results for increasing the deepness of the system identification of ARX processes in adaptive tracking. The main result in this paper is also related to the estimation error sequence and to the properties of the driven noise, in particular to the almost sure estimation of its even moments. Consequently, our result leads us to increase our knowledge on the distribution of the driven noise of a controllable $\operatorname{ARX}(p, q)$ process.

The paper in organized as follows. Section 2 is devoted to the one-dimensional $\operatorname{ARX}(p, q)$ processes in adaptive tracking, while the ASCLT for the least squares estimator is given in Section 3. Our theoretical results are illustrated by numerical experiments in Section 4. A short conclusion is given in Section 5. The almost sure central limit theorem for martingales is provided in Appendix A, while all technical proofs are postponed to Appendix B. 


\section{ARX PROCESSES}

In this section, we focus our attention on the one-dimensional $\operatorname{ARX}(p, q)$ processes in adaptive tracking, given for all $n \geq 0$ by

$$
A(R) X_{n+1}=B(R) U_{n}+\varepsilon_{n+1}
$$

where $R$ stands for the shift-back operator, $X_{n}, U_{n}$ and $\varepsilon_{n}$ are the system output, input and driven noise, respectively. The polynomials $A$ and $B$ are given for all $z \in \mathbb{C}$ by

$$
\begin{aligned}
& A(z)=1-a_{1} z-\cdots-a_{p} z^{p}, \\
& B(z)=1+b_{1} z+\cdots+b_{q} z^{q}
\end{aligned}
$$

where $a_{i}$ and $b_{j}$ are typically unknown real numbers. In all the sequel, we shall make use of the well-known causality assumption on $B$, also known as the minimum phase condition, as well as the usual notion of controllability for one-dimensional ARX processes. To be more precise, we assume that the polynomial $B(z)$ only has zeros with modulus $>1$ and that polynomials $A(z)-1$ and $B(z)$ are coprime. Relation (2.1) may be rewritten in the compact form

$$
X_{n+1}=\theta^{T} \Phi_{n}+U_{n}+\varepsilon_{n+1}
$$

where $\theta^{T}=\left(a_{1}, \ldots, a_{p}, b_{1}, \ldots, b_{q}\right)$ and $\Phi_{n}^{T}=\left(X_{n}^{p}, U_{n-1}^{q}\right)$ with $X_{n}^{p}=\left(X_{n}, \ldots, X_{n-p+1}\right)$ and $U_{n}^{q}=\left(U_{n}, \ldots, U_{n-q+1}\right)$. We shall assume that the driven noise $\left(\varepsilon_{n}\right)$ is a martingale difference sequence adapted to the filtration $\mathbb{F}=\left(\mathcal{F}_{n}\right)$ where $\mathcal{F}_{n}$ is for the $\sigma$-algebra of the events occurring up to time $n$, which means that for all $n \geq 0$, $\mathbb{E}\left[\varepsilon_{n+1} \mid \mathcal{F}_{n}\right]=0$ a.s. Moreover, we assume that, for all $n \geq 0, \mathbb{E}\left[\varepsilon_{n+1}^{2} \mid \mathcal{F}_{n}\right]=\sigma^{2}$ a.s. where $\sigma^{2}>0$. Finally, we assume that $\left(\varepsilon_{n}\right)$ satisfies, for some integer $m \geq 1$ and some real number $a>2 m$,

$$
\sup _{n \geq 0} \mathbb{E}\left[\left|\varepsilon_{n+1}\right|^{a} \mid \mathcal{F}_{n}\right]<\infty \quad \text { a.s. }
$$

The goal of adaptive tracking is to regulate the dynamics of the process $\left(X_{n}\right)$ by forcing the output $X_{n}$ to track, step by step, a predictable reference trajectory $\left(x_{n}\right)$ such that

$$
\sum_{k=1}^{n} x_{k}^{2}=o(n) \quad \text { a.s. }
$$

Moreover, at the same time, we shall also estimate the unknown parameter $\theta$.

First, we focus our attention on the estimation of $\theta$. We shall make use of the least squares estimator which satisfies, for all $n \geq 0$,

$$
\begin{gathered}
\widehat{\theta}_{n+1}=\widehat{\theta}_{n}+S_{n}^{-1} \Phi_{n}\left(X_{n+1}-U_{n}-\widehat{\theta}_{n}^{T} \Phi_{n}\right), \\
S_{n}=\sum_{k=0}^{n} \Phi_{k} \Phi_{k}^{T}+I_{\delta}
\end{gathered}
$$

where the initial value $\hat{\theta}_{0}$ may be arbitrarily chosen and $I_{\delta}$ is the identity matrix of order $\delta=p+q$. 
Next, we are concerned with the choice of the adaptive control sequence $\left(U_{n}\right)$. With that aim, we shall make use of the adaptive tracking control proposed by Aström and Wittenmark [2] given, for all $n \geq 0$, by

$$
U_{n}=x_{n+1}-\widehat{\theta}_{n}^{T} \Phi_{n} .
$$

By substituting (2.5) into (2.2), we obtain the closed-loop system

$$
X_{n+1}-x_{n+1}=\pi_{n}+\varepsilon_{n+1},
$$

where prediction error $\pi_{n}=\left(\theta-\widehat{\theta}_{n}\right)^{T} \Phi_{n}$. Finally, for each integer $m \geq 1$ (this integer will be related to the conditional moments of order $2 m$ of the driven noise), denote by $\left(C_{n}(m)\right)$ and $\left(G_{n}(m)\right)$ the sequences of average costs and estimation errors given by

$$
C_{n}(m)=\frac{1}{n} \sum_{k=1}^{n}\left(X_{k}-x_{k}\right)^{2 m}
$$

and

$$
G_{n}(m)=\sum_{k=1}^{n} k^{m-1}\left\|\widehat{\theta}_{k}-\theta\right\|^{2 m} .
$$

We assume that the polynomial $B(z)$ only has zeros with modulus $>1$. If $r>1$ is strictly less than the smallest modulus of the zeros of $B(z)$, then $B(z)$ is invertible in the ball with center zero and radius $r$ and $B^{-1}(z)$ is a holomorphic function. For all $z \in \mathbb{C}$ such that $|z| \leq r$, denote

$$
P(z)=B^{-1}(z)(A(z)-1)=\sum_{k=1}^{\infty} p_{k} z^{k} .
$$

All the coefficients $p_{k}$ may be explicitly calculated as functions of $a_{1}, \ldots, a_{p}$ and $b_{1}, \ldots, b_{q}[6]$. For any $1 \leq i \leq q$, let

$$
h_{i}=\sum_{k=i}^{\infty} p_{k} p_{k-i+1} .
$$

In addition, denote by $H$ the square matrix of order $q$,

$$
H=\left(\begin{array}{ccccc}
h_{1} & h_{2} & \cdots & h_{q-1} & h_{q} \\
h_{2} & h_{1} & h_{2} & \cdots & h_{q-1} \\
\cdots & \cdots & \cdots & \cdots & \cdots \\
h_{q-1} & \cdots & h_{2} & h_{1} & h_{2} \\
h_{q} & h_{q-1} & \cdots & h_{2} & h_{1}
\end{array}\right)
$$

Let $K$ be the rectangular matrix of dimension $q \times p$ given, if $p \geq q$, by 
while, if $p \leq q$, by

$$
K=\left(\begin{array}{ccccccc}
0 & p_{1} & p_{2} & \cdots & \cdots & p_{p-2} & p_{p-1} \\
0 & 0 & p_{1} & \cdots & \cdots & p_{p-3} & p_{p-2} \\
\cdots & \cdots & \cdots & \cdots & \cdots & \cdots & \cdots \\
0 & \cdots & 0 & p_{1} & p_{2} & \cdots & p_{p-q+1} \\
0 & \cdots & 0 & 0 & p_{1} & \cdots & p_{p-q}
\end{array}\right)
$$

$$
K=\left(\begin{array}{ccccc}
0 & p_{1} & \cdots & p_{p-2} & p_{p-1} \\
0 & 0 & p_{1} & \cdots & p_{p-2} \\
\cdots & \cdots & \cdots & \cdots & \cdots \\
0 & \cdots & 0 & 0 & p_{1} \\
\cdots & \cdots & \cdots & \cdots & \cdots \\
0 & 0 & \cdots & 0 & 0
\end{array}\right)
$$

Finally, denote by $L$ the square matrix of order $\delta=p+q$

$$
L=\left(\begin{array}{cc}
I_{p} & K^{T} \\
K & H
\end{array}\right)
$$

\section{MAIN RESULTS}

Our first result deals with the ASCLT for the least squares estimator $\widehat{\theta}_{n}$ of the unknown parameter $\theta$.

Theorem 3.1. Assume that the ARX $(p, q)$ process is causal and controllable. Moreover, assume that for some real number $a>2$,

$$
\sup _{n \geq 0} \mathbb{E}\left[\left|\varepsilon_{n+1}\right|^{a} \mid \mathcal{F}_{n}\right]<\infty \quad \text { a.s. }
$$

Then, we have the ASCLT

$$
\frac{1}{\log n} \sum_{k=1}^{n} \frac{1}{k} \delta_{\sqrt{k}\left(\widehat{\theta}_{k}-\theta\right)} \Rightarrow \mathcal{N}_{\delta}\left(0, L^{-1}\right) \quad \text { a.s. }
$$

In other words, for any bounded continuous function $h$,

$$
\lim _{n \rightarrow \infty} \frac{1}{\log n} \sum_{k=1}^{n} \frac{1}{k} h\left(\sqrt{k}\left(\widehat{\theta}_{k}-\theta\right)\right)=\int_{\mathbb{R}^{\delta}} h(x) d G(x) \quad \text { a.s. }
$$

where $G$ stands for the $\mathcal{N}_{\delta}\left(0, L^{-1}\right)$ Gaussian measure.

Remark 3.1. One can observe that the scalar variance $\sigma^{2}$ vanishes in the ASCLT.

Corollary 3.1. Assume that the $A R X(p, q)$ process is causal and controllable. Moreover, assume that $\left(\varepsilon_{n}\right)$ satisfies, for some integer $m \geq 1$, condition (2.3). Then, we have

$$
\lim _{n \rightarrow \infty} \frac{1}{\log n} \sum_{k=1}^{n} k^{m-1}\left(\left(\widehat{\theta}_{k}-\theta\right)^{T} L\left(\widehat{\theta}_{k}-\theta\right)\right)^{m}=\ell(m) \quad \text { a.s. }
$$


where

$$
\ell(m)=(p+q) \prod_{k=1}^{m-1}(p+q+2 k) .
$$

Remark 3.2. In the special case $m=1$, we can deduce from (3.3) the quadratic strong law

$$
\lim _{n \rightarrow \infty} \frac{1}{\log n} \sum_{k=1}^{n}\left(\widehat{\theta}_{k}-\theta\right)^{T} L\left(\widehat{\theta}_{k}-\theta\right)=p+q \quad \text { a.s. }
$$

We now focus our attention on the average costs and estimation errors sequences $\left(C_{n}(m)\right)$ and $\left(G_{n}(m)\right)$ given by (2.7) and (2.8). First of all, it was proven in Lemma 3 of [6] that the matrix $L$ is positive definite. Hence, (3.3) immediately implies that

$$
G_{n}(m)=\mathcal{O}(\log n) \quad \text { a.s. }
$$

Furthermore, denote

$$
\Gamma_{n}(m)=\frac{1}{n} \sum_{k=1}^{n} \varepsilon_{k}^{2 m} .
$$

The asymptotic behavior of $\left(C_{n}(m)\right)$ is as follows.

Corollary 3.2. Assume that the $A R X(p, q)$ process is causal and controllable. Moreover, assume that $\left(\varepsilon_{n}\right)$ satisfies, for some integer $m \geq 1$, condition (2.3). Suppose that it exists some integer $1 \leq s \leq m$ such that $\mathbb{E}\left[\varepsilon_{n+1}^{2 s} \mid \mathcal{F}_{n}\right]=\sigma(2 s)$ a.s. Then, $C_{n}(s)$ is a strongly consistent estimator of $\sigma(2 s)$,

$$
\lim _{n \rightarrow \infty} C_{n}(s)=\sigma(2 s) \quad \text { a.s. }
$$

More precisely, for all $0<b<1$ such that $2 m<a b$, we have

$$
\left(C_{n}(s)-\Gamma_{n}(s)\right)^{2}=o\left(n^{b-1}\right) \quad \text { a.s. }
$$

\section{Numerical Experiments}

We provide now some numerical experiments in order to illustrate the most relevant almost sure results of Section 3. More precisely, we shall focus attention on the quadratic strong law given by (3.5) as well as on the almost sure convergence of even moments given by convergence (3.6) for different values of $m$. For the sake of simplicity, we assume that the driven noise $\left(\varepsilon_{n}\right)$ is a sequence of independent and identically distributed random variables sharing the same $\mathcal{N}\left(0, \sigma^{2}\right)$ distribution with $\sigma^{2}=0.8$, and the reference trajectory $\left(x_{n}\right)$ is identically zero. Consider the $\operatorname{ARX}(2,2)$ process given by (2.1) where

$$
A(z)=1+\frac{6}{5} z-\frac{1}{2} z^{2} \quad \text { and } \quad B(z)=1+\frac{2}{5} z+\frac{1}{4} z^{2} .
$$

One may observe that $B$ is causal since its complex roots have modulus 2 . Moreover, one may easily check that the process is controllable and the matrix $L$ is given by 


$$
L=\left(\begin{array}{cccc}
1 & 0 & 0 & 0 \\
0 & 1 & 6 / 5 & 0 \\
0 & 6 / 5 & 244 / 99 & -628 / 495 \\
0 & 0 & -628 / 495 & 244 / 99
\end{array}\right)
$$

In order to illustrate the quadratic strong law given by (3.5), the sample size will increases from $n=100$ to $n=5000$, and we shall denote by $\Delta_{n}$ the average of $N=100$ values of

$$
\frac{1}{\log n} \sum_{k=1}^{n}\left(\widehat{\theta}_{k}-\theta\right)^{T} L\left(\widehat{\theta}_{k}-\theta\right) .
$$

We may conclude by observing Table 1 that, even with the slow growth of the logarithmic function, relative errors are small, and the quadratic strong law is nicely shown. We recall here that the almost sure limit given by (3.5) is $p+q=4$.

\begin{tabular}{|c|c|c|}
\hline$n$ & $\Delta_{n}$ & relative error \\
\hline 100 & 4.33 & $8.14 \%$ \\
\hline 500 & 4.14 & $3.51 \%$ \\
\hline 1000 & 3.97 & $0.69 \%$ \\
\hline 2000 & 4.03 & $0.78 \%$ \\
\hline 5000 & 3.972 & $0.70 \%$ \\
\hline
\end{tabular}

Table 1. Quadratic strong law.

Let us deal now with the almost sure convergence of even moments given by convergence (3.6). For that purpose, we shall consider the average of $N=100$ values of sample size $n=10000$ of $C_{n}(m)$. The corresponding results are presented in Table 2 where the values of $m$ increases from 1 to 5 .

\begin{tabular}{|c|c|c|c|}
\hline$m$ & $C_{n}(m)$ & $\sigma(2 m)$ & relative error \\
\hline 1 & 0.6426 & 0.64 & $0.41 \%$ \\
\hline 2 & 1.245 & 1.229 & $1.84 \%$ \\
\hline 3 & 4.07 & 3.932 & $3.51 \%$ \\
\hline 4 & 18.81 & 17.62 & $6.75 \%$ \\
\hline 5 & 110.61 & 101.47 & $9.00 \%$ \\
\hline
\end{tabular}

Table 2. Convergence of even moments.

We observe that as the value of $m$ increases, the relative error also increases. In other words, it is necessary to take large sample sizes in order to estimate large order even moments. For example, choose the value of $m=5$ and consider the large sample sizes $n=20000,30000$ and 50000 as indicated in Table 3. As expected, for large sample sizes values, the almost sure convergence of even moments can be improved substantially. 


\begin{tabular}{|c|c|c|}
\hline $\mathrm{n}$ & $C_{n}(5)$ & relative error \\
\hline 20000 & 108.54 & $6.90 \%$ \\
\hline 30000 & 106.15 & $4.61 \%$ \\
\hline 50000 & 105.44 & $3.91 \%$ \\
\hline
\end{tabular}

Table 3. Estimation of $\sigma(10)$.

\section{Conclusion}

In this paper, we established the almost sure central limit theorem for the least squares estimator of the unknown parameter of a controllable $\operatorname{ARX}(p, q)$ process in adaptive tracking. We have also provided a strongly consistent estimator for the even moments of the driven noise. Even when most of the engineering methods do not consider high order moments, it is useful to go deeper into the knowledge of the driven noise distribution through the estimation of such moments since it gives us a better notion of the underlying uncertainty.

\section{ApPENDix A}

\section{On THE ALMOST SURE CENTRAL LIMIT THEOREM FOR MARTINGALES}

The goal of this Appendix is to highlight the ASCLT for martingales [3, 4, 5, 15, 16, 26] to the control community. Let $(\Omega, \mathcal{F}, \mathbb{P})$ be a probability space endowed with a filtration $\mathbb{F}=\left(\mathcal{F}_{n}\right)$ where $\mathcal{F}_{n}$ is for the $\sigma$-algebra of the events occurring up to time $n$. Assume that $\left(M_{n}\right)$ be a sequence of integrable random vectors in $\mathbb{R}^{d}$ such that, for all $n \geq 0, M_{n}$ is $\mathcal{F}_{n}$-measurable. We shall say that $\left(M_{n}\right)$ is a martingale with respect to the filtration $\mathbb{F}$ if for all $n \geq 0, \mathbb{E}\left[M_{n+1} \mid \mathcal{F}_{n}\right]=M_{n}$ almost surely. Throughout this Appendix, $\left(\varepsilon_{n}\right)$ is a martingale difference sequence adapted to $\mathbb{F}$ such that, for all $n \geq 0, \mathbb{E}\left[\varepsilon_{n+1}^{2} \mid \mathcal{F}_{n}\right]=\sigma^{2}$ a.s. where $\sigma^{2}>0$. Let $\left(\Phi_{n}\right)$ be a sequence of random vectors of $\mathbb{R}^{d}$, adapted to $\mathbb{F}$. Denote by $\left(M_{n}\right)$ the locally square integrable martingale

$$
M_{n}=M_{0}+\sum_{k=1}^{n} \Phi_{k-1} \varepsilon_{k},
$$

where the initial value $M_{0}$ can be taken arbitrarily. Its increasing process $\langle M\rangle_{n}$ is defined, for all $n \geq 1$, by

$$
\langle M\rangle_{n}=\sum_{k=1}^{n} \mathbb{E}\left[\Delta M_{k} \Delta M_{k}^{T} \mid \mathcal{F}_{k-1}\right]
$$

where $\Delta M_{k}=M_{k}-M_{k-1}$. We clearly have $\langle M\rangle_{n}=\sigma^{2} S_{n-1}$ where

$$
S_{n}=\sum_{k=0}^{n} \Phi_{k} \Phi_{k}^{T}
$$

A simplified version of the ASCLT for multivariate martingales is as follows [14]. 
Theorem A.1. Assume that it exists a positive definite symmetric matrix $L$ such that

$$
\lim _{n \rightarrow \infty} \frac{1}{n} S_{n}=L \quad \text { a.s. }
$$

Moreover, assume that $\left(M_{n}\right)$ satisfies Lindeberg's condition which means that for all $\varepsilon>0$

$$
\lim _{n \rightarrow \infty} \frac{1}{n} \sum_{k=1}^{n} \mathbb{E}\left[\left\|\Delta M_{k}\right\|^{2} \mathrm{I}_{\left\{\left\|\Delta M_{k}\right\| \geq \varepsilon \sqrt{n}\right\}} \mid \mathcal{F}_{k-1}\right]=0 \quad \text { a.s. }
$$

Then, we have the ASCLT

$$
\frac{1}{\log n} \sum_{k=1}^{n} \frac{1}{k} \delta_{M_{k} / \sqrt{k}} \Rightarrow \mathcal{N}_{d}\left(0, \sigma^{2} L\right) \quad \text { a.s. }
$$

In other words, for any bounded continuous function $h$,

$$
\lim _{n \rightarrow \infty} \frac{1}{\log n} \sum_{k=1}^{n} \frac{1}{k} h\left(\frac{M_{k}}{\sqrt{k}}\right)=\int_{\mathbb{R}^{d}} h(x) d G(x) \quad \text { a.s. }
$$

where $G$ stands for the $\mathcal{N}_{d}\left(0, \sigma^{2} L\right)$ Gaussian measure.

The convergence of the even moments in the ASCLT for multivariate martingales was established in [5].

Theorem A.2. Assume that the almost sure convergence (A.1) is satisfied. In addition, suppose that for some integer $m \geq 1$ and for some real number $a>2 m$,

$$
\sup _{n \geq 0} \mathbb{E}\left[\left|\varepsilon_{n+1}\right|^{a} \mid \mathcal{F}_{n}\right]<\infty \quad \text { a.s. }
$$

Then, we have

$$
\lim _{n \rightarrow \infty} \frac{1}{\log n} \sum_{k=1}^{n} \frac{1}{k}\left(M_{k}^{T} S_{k-1}^{-1} M_{k}\right)^{m}=\ell(m) \quad \text { a.s. }
$$

where

$$
\ell(m)=d \sigma^{2 m} \prod_{k=1}^{m-1}(d+2 k)
$$

Remark A.1. The limit $\ell(m)$ corresponds exactly to the mean value of $\|Z\|^{2 m}$ where $Z$ has a standard $\mathcal{N}_{d}\left(0, \sigma^{2} I_{d}\right)$ distribution. Consequently, Theorem A.2 can be seen as the convergence of moments of order $2 m$ in the ASCLT for multivariate martingales. 


\section{Appendix B}

\section{PROOFS OF OUR MAIN RESULTS}

Proof of Theorem 3.1. It follows from (2.2) and (2.4) that for all $n \geq 1$,

$$
\widehat{\theta}_{n}-\theta=S_{n-1}^{-1} M_{n}
$$

where

$$
M_{n}=M_{0}+\sum_{k=1}^{n} \Phi_{k-1} \varepsilon_{k}
$$

with $M_{0}=\widehat{\theta}_{0}-\theta$. It was proven in Theorem 5 of [6] that

$$
\lim _{n \rightarrow \infty} \frac{S_{n}}{n}=\Lambda \quad \text { a.s. }
$$

where $\Lambda=\sigma^{2} L$ and the limiting matrix $L$ is given by (2.11). Moreover, we can deduce from (3.1) that Lindeberg's condition (A.2) is satisfied. Consequently, we obtain (3.2) from Theorem A.1 together with (B.1) and (B.2).

Proof of Corollary 3.1. The almost sure convergence (3.3) follows from the conjunction of Theorem 3.1 together with (B.1) and (B.2), using the same arguments as in the proof of Corollary 3.3 in [5].

Proof of Corollary 3.2. For any integer $1 \leq s \leq m$, we obtain from (2.6) that

$$
\begin{aligned}
n\left(C_{n}(s)-\Gamma_{n}(s)\right) & =\sum_{k=1}^{n}\left(X_{k}-x_{k}\right)^{2 s}-\sum_{k=1}^{n} \varepsilon_{k}^{2 s} \\
& =\sum_{k=1}^{n}\left(\pi_{k-1}+\varepsilon_{k}\right)^{2 s}-\sum_{k=1}^{n} \varepsilon_{k}^{2 s} \\
& =\sum_{k=1}^{n} \pi_{k-1}^{2 s}+R_{n}(s)
\end{aligned}
$$

where

$$
R_{n}(s)=\sum_{l=1}^{2 s-1} \sum_{k=1}^{n}\left(\begin{array}{c}
2 s \\
l
\end{array}\right) \pi_{k-1}^{2 s-l} \varepsilon_{k}^{l} .
$$

On the one hand, in the special case $s=1$, we deduce from Theorem A.2 with $m=1$ that

$$
\lim _{n \rightarrow \infty} \frac{1}{\log n} \sum_{k=1}^{n} \pi_{k}^{2}=\sigma^{2}(p+q) \quad \text { a.s. }
$$

In addition, we find from the strong law of large numbers for martingales [18] that

$$
R_{n}(1)=o(\log n) \quad \text { a.s. }
$$

Hence, we obtain from (B.3) together with (B.4) and (B.5) that

$$
\lim _{n \rightarrow \infty} \frac{n}{\log n}\left(C_{n}(1)-\Gamma_{n}(1)\right)=\sigma^{2}(p+q) \quad \text { a.s. }
$$


Therefore, as

$$
\lim _{n \rightarrow \infty} \Gamma_{n}(1)=\sigma^{2} \quad \text { a.s. }
$$

convergence (B.6) clearly leads to (3.6) since $\sigma(2)=\sigma^{2}$. On the other hand, for $2 \leq s \leq m$, it follows from convergence (B.2) that $\log d_{n} \sim(p+q) \log n$ where $d_{n}=\operatorname{det}\left(S_{n}\right)$. Consequently, Corollary 3.1 of [5] lead us to

$$
\lim _{n \rightarrow \infty} \frac{1}{\log n} \sum_{k=1}^{n} \pi_{k}^{2 s}=0 \quad \text { a.s. }
$$

Moreover, it is not hard to see that the remainder term $R_{n}(s)$ plays a negligible role. Hence, as

$$
\lim _{n \rightarrow \infty} \Gamma_{n}(s)=\sigma(2 s) \quad \text { a.s. }
$$

we obtain (3.6) from (B.3) and (B.7). Finally, we deduce (3.7) from Remark 3.1 of [5], which completes the proof of Corollary 3.2 .

\section{REFERENCES}

[1] K. J. Aström and P. Eykhoff, System identification-A survey, Automatica, Vol. 7, pp. 123-162, 1971.

[2] K. J. Aström and B. Wittenmark, Adaptive Control, Addison-Wesley, New York, 1995.

[3] B. Bercu, On the convergence of moments in the almost sure central limit theorem for martingales with statistical applications, Stoch. Process. Their Appl., Vol. 111, pp. 157-173, 2004.

[4] B. Bercu and J. C. Fort, A moment approach for the almost sure central limit theorem for martingales, Studia Sci. Math. Hung., Vol. 45, pp. 139-159, 2008.

[5] B. Bercu, P. Cenac, G. Fayolle, On the almost sure central limit theorem for vector martingales : convergence of moments and statistical applications, J. Appl. Probab., Vol. 46, pp. 151-169, 2009.

[6] B. Bercu and V. Vázquez, A new concept of strong controllability via the Schur complement in adapative tracking, Automatica, Vol. 46, pp. 1799-1805, 2010.

[7] B. Bercu and V. Vázquez. On the usefulness of persistent excitation in ARX adaptive tracking, Int. J. Control, Vol. 83, pp. 1145-1154, 2010.

[8] B. Bercu, B. Portier and V. Vázquez, On the asymptotic behavior of the Durbin-Watson statistic for ARX processes in adaptive tracking, Int. J. Adapt. Control Signal Process., Vol. 28, pp. 1002-1023, 2014.

[9] B. Bercu, B. Portier and V. Vázquez, A Durbin-Watson serial correlation test for ARX processes via excited adaptive tracking, Int. J. Control, Vol. 88, Issue 12, pp. 2611-2618, 2015.

[10] I. Berkes and E. Csáki, A universal result in almost sure central limit theory, Stoch. Process. Their Appl., Vol. 94, pp. 105-134, 2001.

[11] T. Bin, P. Zuoxiang and S. Nadarajah, An extension of almost sure central limit theorem for order statistics, Extremes Vol. 12, pp. 201-209, 2009.

[12] G. A. Brosamler, An almost everywhere central limit theorem, Math. Proc. Cambridge Philos. Soc., Vol. 104, pp. 561-574, 1988.

[13] P. Cenac, On the convergence of moments in the almost sure central limit theorem for stochastic approximation algorithms, ESAIM Probab. Stat., Vol. 17, pp. 179-194, 2013.

[14] F. Chaâbane, F. Maâouia, and A. Touati, Généralisation du théorème de la limite centrale presque-sûr pour les martingales vectorielles, C. R. Acad. Sci. Paris Sér. I Math., Vol. 326, pp. 229-232, 1998.

[15] F. Chaâbane and F. Maâouia, Théorèmes limites avec poids pour les martingales vectorielles, ESAIM Probab. Statist., Vol. 4, pp. 137-189, 2000. 
[16] F. Chaâbane, Invariance principles with logarithmic averaging for martingales, Studia Sci. Math. Hung., Vol. 37, pp. 21-52, 2001.

[17] N. Cho, H. S. Shin, Y. Kim and A. Tsourdos, Composite model reference adaptive control with parameter convergence under finite excitation, IEEE Trans. Autom. Control, Vol. 63, Issue 3, pp. 811-818, 2018.

[18] M. Duflo, Random iterative models, Vol. 34 of Applications of Mathematics. Springer-Verlag, Berlin, 1997.

[19] Y. F. Gao, C. Wen, W. Wang, Adaptive tracking control for a class of stochastic nonlinear systems with input saturation, IEEE Trans. Automat. Control, Vol. 62, pp. 2498-2504, 2017.

[20] M. Gevers, System Identification without Lennart Ljung: what would have been different?, Forever Ljung in System Identification, Studentlitteratur, 2006.

[21] A. Heydari, Stability analysis of optimal adaptive control using value iteration with approximation errors, IEEE Trans. Autom. Control (Early access), 2018.

[22] H. Holzmann, S. Koch, A. Min, Almost sure limit theorems for U-statistics, Statist. Probab. Letters, Vol. 69, pp. 261-269, 2004.

[23] X. Hong, R. J. Mitchell, S. Chen, C. J. Harris, K. Li and G. W. Irwin, Model selection approaches for non-linear system identification: a review, International Journal of Systems Science, Vol. 39, pp. 925-946, 2008.

[24] F. Jaramillo-Lopez, G. Kenne and F. Lamnabhi-Lagarrigue, Adaptive control for a class of uncertain nonlinear systems: Application to photovoltaic control systems, IEEE Trans. Autom. Control, Vol. 62, pp. 393-398, 2017.

[25] M. T. Lacey and W. Phillip, A note on the almost sure central limit theorem, Statist. Probab. Letters, Vol. 9, pp. 201-205, 1990.

[26] M. A. Lifshits, Almost sure limit theorem for martingales, In Limit theorems in probability and statistics, János Bolyai Math. Soc., pp. 367-390, 2002.

[27] L. Ljung, Adaptation and tracking in system identification - a survey, Automatica, Vol. 26, pp. 7-21, 1990 .

[28] M. Pelletier, An almost sure central limit theorem for stochastic approximation algorithms, J. Multivariate Anal., Vol 71, pp. 76-93, 1999.

[29] G. Pillonetto, F. Dinuzzo, T. Chen, G. De Nicolao, L. Ljung, Kernel methods in system identification, machine learning and function estimation: A survey, Automatica, Vol. 50, pp. 657682, 2014 .

[30] P. Schatte, On strong versions of the central limit theorem, Math. Nachr., Vol. 137, pp. 249-256, 1988.

[31] G. Tao and G. Song, Higher-order tracking properties of model reference adaptive control systems, IEEE Trans. Autom. Control (Early Access), 2018.

[32] L. A. Zadeh, From Circuit Theory to System Theory, Proceedings of the IRE, Vol. 50, pp. 856-865, 1962.

[33] Y. Zhao, J. Guo and J.F. Zhang, Adaptive tracking control of linear systems with binaryvalued observations and periodic target, IEEE Trans. Autom. Control, Vol. 58, pp. 1293-1298, 2013.

[34] B. Zhu and X. Xia, Adaptive model predictive control for unconstrained discrete-time linear systems with parametric uncertainties, IEEE Trans. Autom. Control, Vol. 61, pp. 3171-3176, 2016.

E-mail address: bernard.bercu@u-bordeaux.fr

E-mail address: vvazquez@fcfm.buap.mx

Université de Bordeaux, Institut de Mathématiques, UMR 5251, 351 COURS DE LA LibÉration, 33405 TAlence CEDEX, France.

Universidad Autónoma de Puebla, Facultad de Ciencias Fisico Matemáticas, Avenida San Claudio y Rio Verde, 72570 Puebla, Mexico. 